\title{
Peritumoral lymphatic microvessel density is related to poor prognosis in lung adenocarcinoma: A retrospective study of 65 cases
}

\author{
BI-CHENG ZHANG ${ }^{*}$, SHA GUAN* ${ }^{*}$ YA-FEI ZHANG, GUO-QING YAO, \\ BO YANG, YONG ZHAO, ZHI-GUO RAO and JIAN-FEI GAO \\ Department of Oncology, Wuhan General Hospital of Guangzhou Command, \\ People's Liberation Army, Wuhan 430070, P.R. China
}

Received November 10, 2011; Accepted January 19, 2012

DOI: $10.3892 /$ etm.2012.470

\begin{abstract}
Although recent investigations have identified that lymphangiogenesis is associated with regional lymph node metastasis and tumor prognosis in non-small cell lung cancer (NSCLC), peritumoral lymphatic microvessel density (LMVD) and its prognostic significance in lung adenocarcinoma remain unknown. In the present study, we assessed peritumoral LMVD in lung adenocarcinoma and investigated its correlation with patient prognosis. Using immunohistochemistry (SP method), the D2-40-positive peritumoral LMVD count in lung adenocarcinoma was found to be $11.56 \pm 10.73$, which was higher than intratumoral LMVD ( $\mathrm{P}<0.001)$, and was found to be associated with lymphatic metastasis $(\mathrm{P}=0.003)$ and $\mathrm{pTNM}$ staging $(\mathrm{P}=0.046)$. Furthermore, a significant difference in the patient overall survival time was demonstrated between tumors with a high peritumoral LMVD and those with a low peritumoral LMVD $(\mathrm{P}=0.005)$. Finally, using multivariate analysis, it was determined that peritumoral LMVD, lymphatic metastasis and pTNM staging were independent prognostic factors. In conclusion, the results suggest that D2-40-positive peritumoral LMVD may predict the prognosis of lung adenocarcinoma.
\end{abstract}

\section{Introduction}

Lung cancer is the leading cause of tumor-related mortality throughout the world, and adenocarcinoma has surpassed squamous cell carcinoma as the most frequent type of lung cancer (1). Lung adenocarcinoma is well known for its ability to involve metastatic disease even at the early stages of tumor

Correspondence to: Professor Jian-Fei Gao, Department of Oncology, Wuhan General Hospital of Guangzhou Command, People's Liberation Army, Wuhan 430070, P.R. China

E-mail: jianfeigao1957@hotmail.com

*Contributed equally

Key words: lymphatic microvessel density, lymphangiogenesis, lung adenocarcinoma, prognosis growth, which generally results in treatment failure. Due to the early acquisition of a metastatic phenotype and the associated poor prognosis of lung adenocarcinoma, investigation into the molecular mechanisms responsible for metastasis may lead to the development of new treatments for these patients.

Regional lymph node metastasis is a major route for tumor metastasis in non-small cell lung cancer (NSCLC), which is also one of the most significant prognostic indicators for NSCLC patients. Lymphangiogenesis is considered to be the initial step and key event of lymphatic and regional lymph node metastasis, but only peritumoral lymphangiogenesis is functional $(2,3)$. A number of studies have provided support for the contribution of vascular endothelial growth factor (VEGF)-C and VEGF-D, and their respective receptors, including vascular endothelial growth factor receptor (VEGFR)-2 and VEGFR-3, in tumor-induced lymphangiogenesis $(4,5)$. Not only tumor cells, but also inflammatory cells in tumor stroma including tumor-associated macrophages (TAMs), express VEGF-C and/ or VEGF-D, and induce peritumoral lymphangiogenesis and lymph node metastasis (6-8). Lymphatic microvessel density (LMVD) is a significant indicator of tumor lymphangiogenesis. A number of studies have demonstrated that LMVD is an independent prognostic factor in NSCLC (9-12). However, no significant association between LMVD and lymph node metastasis in NSCLC has been reported (13). Thus, the correlation of lymphangiogenesis with lymph node metastasis and patient survival is controversial and remains to be clarified. Moreover, no study has examined whether the prognosis of patients with lung adenocarcinoma is correlated with the peritumoral LMVD rather than the intratumoral LMVD.

This study investigated the immunohistochemically determined count of LMVD and found that peritumoral lymphangiogenesis is related to poor prognosis in patients with lung adenocarcinoma.

\section{Materials and methods}

Patients and tissue samples. A total of 65 patients with lung adenocarcinoma (38 male and 27 females; mean age, 51.5 years; age range, 32-76 years) who underwent either lobectomy or pneumonectomy at Wuhan General Hospital of 
Table I. Correlation between D2-40 positive LMVD and clinicopathological features (mean $\pm \mathrm{SD}$ ).

\begin{tabular}{lccc}
\hline $\begin{array}{l}\text { Clinicopathological } \\
\text { features }\end{array}$ & $\mathrm{n}$ & $\begin{array}{c}\text { Intratumoral } \\
\text { LMVD }\end{array}$ & $\begin{array}{c}\text { Peritumoral } \\
\text { LMVD }\end{array}$ \\
\hline $\begin{array}{l}\text { Gender } \\
\text { Male }\end{array}$ & 38 & $4.02 \pm 2.11$ & $11.02 \pm 10.29$ \\
$\quad$ Female & 27 & $3.95 \pm 1.73$ & $12.81 \pm 11.23$ \\
Age & & & \\
$\geq 55$ years & 39 & $3.98 \pm 2.10$ & $12.98 \pm 11.04$ \\
$\quad<55$ years & 26 & $4.15 \pm 1.26$ & $10.99 \pm 9.61$ \\
$\quad \begin{array}{l}\text { Differentiation } \\
\text { Favorable and intermediate }\end{array}$ & 45 & $4.13 \pm 1.96$ & $11.95 \pm 10.43$ \\
$\quad$ Poor & 20 & $3.98 \pm 2.16$ & $10.90 \pm 10.17$ \\
$\quad$ Lymphatic metastasis & & & \\
$\quad \begin{array}{l}\text { Positive } \\
\text { Negative }\end{array}$ & 36 & $3.12 \pm 2.56$ & $14.98 \pm 5.60^{\mathrm{a}}$ \\
pTNM stage & 29 & $4.86 \pm 2.27$ & $9.91 \pm 2.16$ \\
$\quad$ I and II & & & \\
III and IV & 38 & $4.31 \pm 2.72$ & $13.54 \pm 10.32^{\mathrm{a}}$ \\
\hline
\end{tabular}

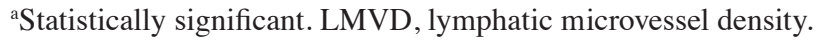

Guangzhou Command, People's Liberation Army, China, were investigated. The patients underwent tumor resection between 2003 and 2006. None of the patients received any preoperative chemotherapy or radiotherapy. The lesions of the 65 patients were staged according to the UICC 2010 pTNM classification (7th edition), and stage I, II, III and IV lesions were present in $14,24,25$ and 2 patients, respectively. Histologically, according to the new classification proposed for lung adenocarcinoma by the IASLC/ATS/ERS 2011 (14), 16 tumors were graded as favorably differentiated (non-mucinous lepidic), 29 as intermediately differentiated (papillary and acinar) and 20 as poorly differentiated (solid and micropapillary) adenocarcinoma. Lymph node metastasis occurred in 36 patients, while the other 29 patients had no lymph node metastasis. All patients accepted complete removal of the tumor and were treated by standardized therapy following surgery. All patients were followed up and their outcomes were known. The clinicopathological parameters of those patients with lung adenocarcinoma are shown in Table I.

LMVD by immunohistochemistry. D2-40 was used as the immunohistochemical marker for lymphatic endothelial cells (LECs) for the evaluation of LMVD (15). Resected tissue specimens were fixed in formalin, embedded in paraffin, and cut into 5- $\mu \mathrm{m}$ serial sections. These sections were then deparaffinized in xylene, and rehydrated through graded alcohol and deionized water. The slides were immunostained with a mouse monoclonal antibody D2-40 (1:200; Signet Laboratories, Dedham, MA, USA) at $4^{\circ} \mathrm{C}$ overnight, and subsequently exposed to a biotinylated secondary antibody for $20 \mathrm{~min}$, followed by treatment with streptavidin peroxidase. For color development, the slides were stained with 3,3'diami- nobenzidine (DAB), and counterstained with hematoxylin and eosin (H\&E). A reddish-brown precipitate in the cytoplasm of LECs indicated a positive reaction.

Following scanning of the immunostained sections at low magnification (x100), the regions with the greatest number of distinctly highlighted lymphatic foci (hot spots) were selected by two observers at the same time. The two observers then independently evaluated the slides for the LMVD count using $\times 400$ magnification (field, $0.03 \mathrm{~mm}^{2}$ ) in three regions without knowledge of the tumor status and the stains used. The intratumoral compartment was the area encompassing cancer glands in the H\&E section. The peritumoral compartment was defined as the area around the intratumoral compartment (a 1-mm band including the edge of the tumor and just outside the tumor). Single immunoreactive endothelial cells or endothelial cell clusters separate from other microvessels were counted as a vessel according to previous procedures (6).

Statistical analysis. The intratumoral or peritumoral LMVD was expressed as the mean \pm SD. Statistical differences between the means were analyzed by the independent-samples t-test. On the basis of LMVD, patients were classified into the high or low peritumoral LMVD group, and the overall survival rate was compared between the two groups. Overall survival time was calculated from the date of surgery until mortality or, if the patient was still alive, until the last follow-up visit. Mortality from any cause was considered for overall survival. Two overall survival rates were calculated by the Kaplan-Meier method and compared by the log-rank test. Each prognostic factor was evaluated with regard to survival in a multivariate analysis by the Cox proportional hazards regression model. $\mathrm{P}<0.05$ was considered to indicate a statistically significant difference. All statistical analyses were performed with SPSS 17.0 (SPSS Inc., Chicago, IL, USA).

\section{Results}

D2-40-positive LMVD in lung adenocarcinoma. D2-40-positive lymph vessels were observed in all 65 lung adenocarcinoma samples examined. Staining of lymphatic vessels with characteristic irregular morphology, empty lumina without red blood cells and thin endothelium were strong and distinct when such lymphatic vessels were present. Vessels containing red blood cells were not stained. In lung adenocarcinoma, D2-40-positive vessels were detected more in the peritumoral stroma (Fig. 1A and B) than in the intratumoral compartment (Fig. 1C and D). The count of D2-40-positive peritumoral LMVD was $11.56 \pm 10.73$, which was higher than that of the intratumoral LMVD $(3.96 \pm 1.15)(\mathrm{P}<0.001)$.

Correlation of D2-40-positive LMVD with clinicopathological features. Table I shows the correlation between peritumoral or intratumoral LMVD and clinicopathological features. Peritumoral LMVD was significantly associated with lymph node metastasis $(\mathrm{P}=0.003)$ and pTNM staging $(\mathrm{P}=0.046)$, but not with gender, age and differentiation. Intratumoral LMVD did not correlate with any of the clinicopathological features.

Prognostic significance of D2-40-positive LMVD. To assess the prognostic significance of D2-40-positive peritumoral 

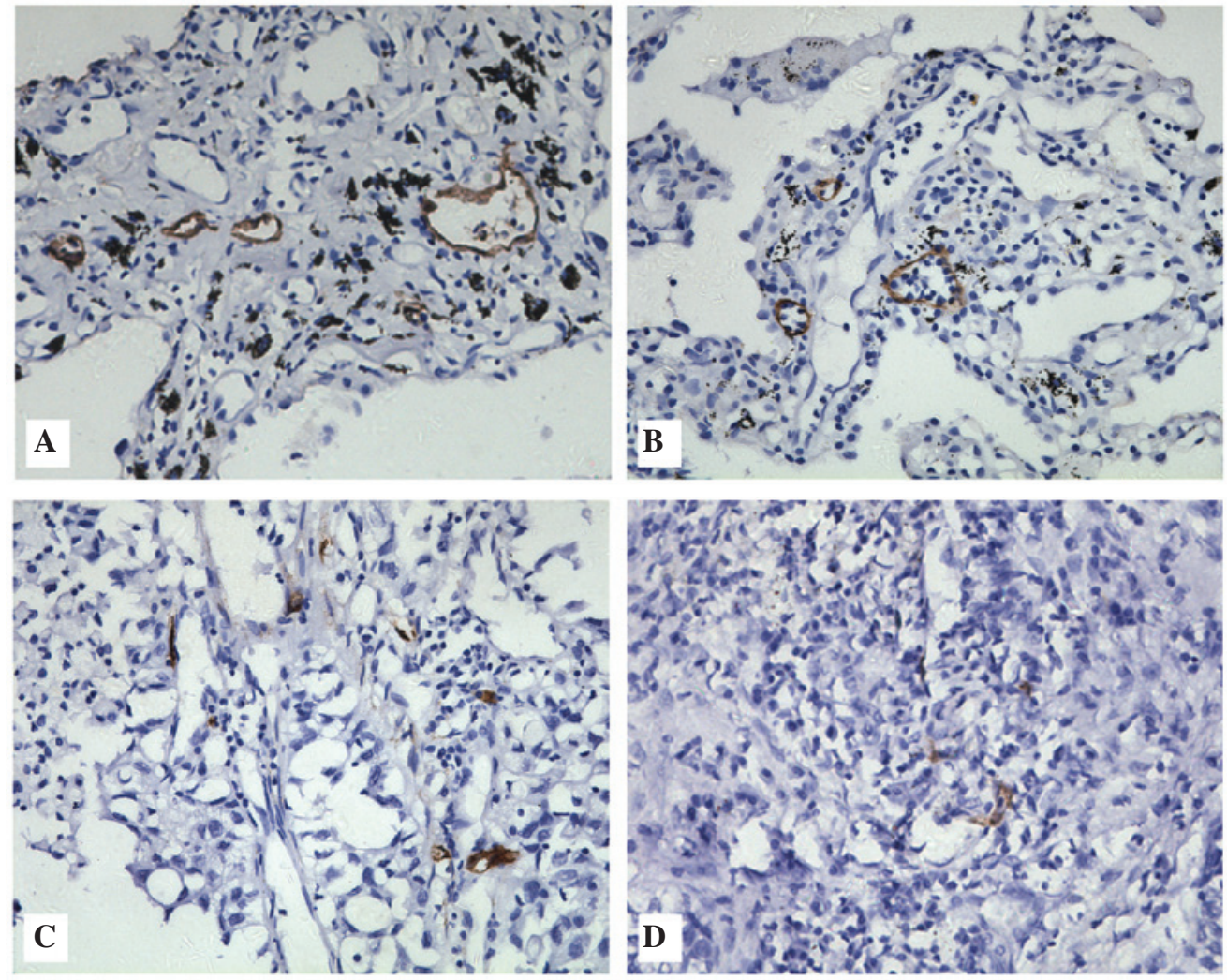

Figure 1. (A and B) Peritumoral LMVD and (C and D) intratumoral LMVD in lung adenocarcinoma samples. D2-40-positive staining was detected in lymph vessels by immunohistochemical staining (magnification, x400; SP method). LMVD, lymphatic microvessel density.

Table II. Multivariate analysis of various prognostic factors in patients.

\begin{tabular}{llrc}
\hline Factor & P-value & HR & $95 \%$ CI \\
\hline Gender & 0.169 & 0.620 & $0.312-1.226$ \\
Age & 0.558 & 1.008 & $0.982-1.033$ \\
Differentiation & 0.655 & 0.852 & $0.422-1.720$ \\
Lymphatic metastasis & $0.040^{\mathrm{a}}$ & 0.470 & $0.229-0.966$ \\
pTNM stages & $0.000^{\mathrm{a}}$ & 24.199 & $4.552-128.6$ \\
Intratumoral LMVD & 0.761 & 0.955 & $0.710-1.285$ \\
Peritumoral LMVD & $0.002^{\mathrm{a}}$ & 1.123 & $1.042-1.210$
\end{tabular}

a Statistically significant. LMVD, lymphatic microvessel density.

LMVD, patients were classified into two groups on the basis of peritumoral LMVD. A median value of 11 was used as the cut-off in peritumoral LMVD. The median overall survival times for the patients with peritumoral LMVD $\leq 11$ or $>11$ were 31 and 13 months, respectively, demonstrating a significant difference in overall survival ( $\mathrm{P}=0.005$ ) (Fig. 2A). Meanwhile, there was a significant difference between the survival rate curve in the positive and negative lymphatic metastasis groups (Fig. 2B) $(\mathrm{P}=0.002)$. Fig. $2 \mathrm{C}$ shows the significant difference between survival rate in stages I and II and stages III and IV $(\mathrm{P}=0.000)$.
Multivariate analysis. Intratumoral LMVD, peritumoral LMVD and other factors, including gender, age, differentiation, lymph node metastasis and pTNM staging, were analyzed by Cox proportional hazards regression models in all 65 patients with lung adenocarcinoma. Peritumoral LMVD, as well as lymph node metastasis and pTNM staging, were independent prognostic factors for overall survival (Table II).

\section{Discussion}

Our data revealed that D2-40 was the most specific lymphatic endothelial marker for the detection of lymphangiogenesis. In recent years, the discovery of LEC markers has facilitated detailed analyses of the nature and structural organization of lymphatic vessels and their growth (lymphangiogenesis). The understanding of the molecular mechanisms of lymphangiogenesis and the elucidation of the development of normal and pathological tissues are expected to lead to the development of therapy for intractable diseases, including malignant tumors and lymphadema. Common LEC markers include VEGFR-3, lymphatic vessel hyaluronan receptor-1 (LYVE-1), Prox-1 and podoplanin (16). Among these, podoplanin is mainly expressed in LECs, keratinocytes, choroid plexus epithelial cells, alveolar cells and few tumor cells. To date no research has reported the assessment of podoplanin in blood capillary. Furthermore, D2-40 is a sialoglycoprotein separated from fetal testiculoma or germinoma, which can be used to detect podoplanin (17). We used all the abovementioned antibodies to detect lymphatic vessels in the preliminary experiment, 
A

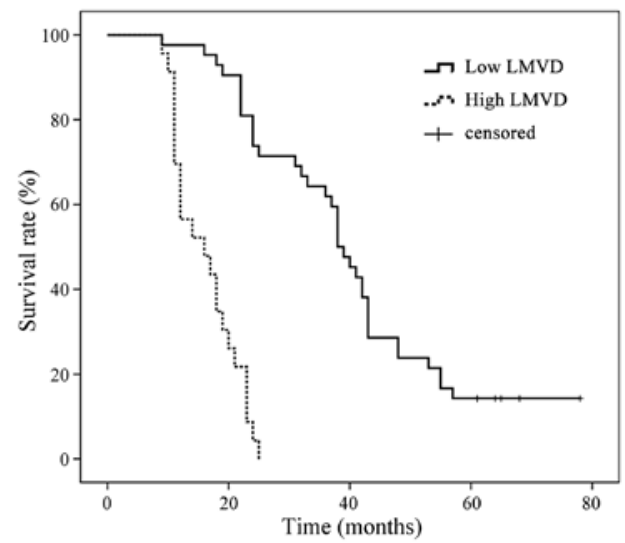

B

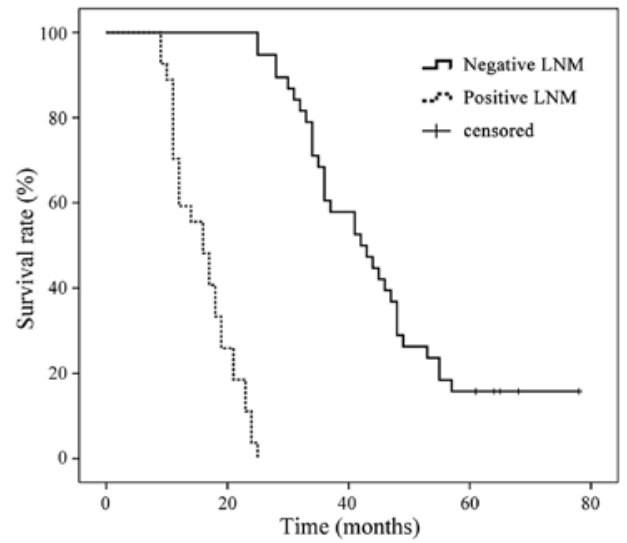

C

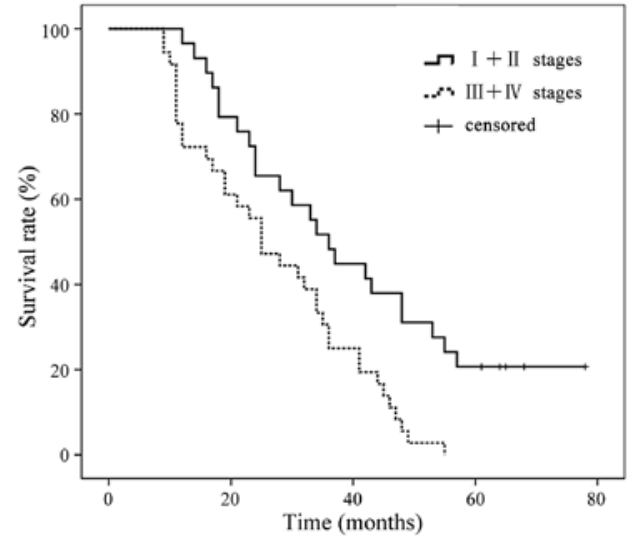

Figure 2. Kaplan-Meier curves for overall survival for (A) D2-40-positive peritumoral LMVD, (B) lymph node metastasis and (C) pTNM staging in patients with lung adenocarcinoma. (A) The difference in overall survival rate between the high and the low peritumoral LMVD group $(\mathrm{P}=0.005)$. (B) The difference in overall survival rate between the positive and the negative lymph node metastasis group $(\mathrm{P}=0.002)$. (C) The difference in overall survival rate between the patients in stages I and II and stages III and IV (P=0.000). LNM, lymph node metastasis; LMVD, lymphatic microvessel density.

and the results demonstrated that no antibody was expressed in $100 \%$ of the LECs (data not shown), and D2-40 was the most specific antibody for the detection, while VEGFR-3 was the least. D2-40 can also be detected in tumor cells, stromal fibroblasts or myofibroblasts (18). In NSCLC, D2-40 immunoreactivity in tumor cells can be used to distinguish between adenocarcinoma and squamous cell carcinoma (19). Therefore, $\mathrm{H} \& \mathrm{E}$ staining combined with a D2-40-positive signal was considered helpful in determining lymphangiogenesis in lung adenocarcinoma.
The data revealed that D2-40-positive peritumoral LMVD in lung adenocarcinoma was higher than intratumoral LMVD. The correlation between LMVD and poor prognosis of tumor patients has been identified in breast cancer, prostate adenocarcinoma, colon cancer, melanoma, NSCLC and oral squamous cell carcinoma (20-24). However, the correlation between LMVD status (intratumoral or peritumoral) and tumor prognosis has not yet been fully clarified. Several studies have demonstrated that functional lymphatics existing in the tumor parenchyma are sufficient for lymphatic metastasis; therefore, high intratumoral LMVD is essential for the metastatic spread and prognosis in squamous cell carcinomas of the head and neck region $(25,26)$. In renal cell carcinoma, intratumoral lymphatics were associated with tumor aggressiveness, and patients with intratumoral lymphatics were found to have poor prognosis (27). However, additional studies demonstrated that intratumoral lymphatic vessels may not be completely functional, as these vessels collapse under high intratumoral pressure (28). We favor the view that local lymphatic vessels at the tumor margin are more vital to the spread of tumor cells. This is achieved through the process of vessel sprouting under the effect of interstitial fluid hypertension and tumor or stroma-secreted VEGF-C/D $(29,30)$. In gastric cancer, increased peritumoral LMVD, but not intratumoral LMVD, was significantly associated with the VEGF-C/-D/VEGFR-3 system, and could be an independent risk factor for lymph node metastasis and a prognostic factor (31). In endometrial carcinoma, peritumoral LMVD was also an independent prognostic factor for progression-free survival and overall survival (32). However, the correlation between intratumoral and peritumoral lymphangiogenesis and the survival of lung adenocarcinoma patients remains unknown.

In the present study, we found that peritumoral, but not intratumoral lymphangiogenesis, played a significant role in the progression and metastasis of lung adenocarcinoma. In a previous study, high LMVD, induced by VEGF-C or VEGF-D expression in cancer cells, was found to be a good indicator of lymphatic metastases and lymphatic vessel invasion in lung adenocarcinoma (33). In the present study, the data did not demonstrate a positive correlation between intratumoral lymphangiogenesis in lung adenocarcinoma and any clinicopathological parameters; therefore, intratumoral LMVD was not a prognostic factor. It was presumed that intratumoral LMVD may be non-functional and may play only a minor role in primary tumor dissemination. A recent study revealed that there was no significant association between peritumoral LMVD and clinicopathological parameters, including lymphatic vessel invasion, lymph node metastasis and survival in lung adenocarcinoma (19). However, in the present study, a positive correlation was found between peritumoral LMVD and lymphatic metastasis or pTNM staging, and high peritumoral LMVD reduced the overall survival of patients. Therefore, peritumoral LMVD, lymphatic metastasis and pTNM stage may be independent risk factors for prognosis. These results demonstrate that peritumoral, but not intratumoral LMVD, may predict the prognosis of lung adenocarcinoma. The contradicting results regarding the role of peritumoral LMVD in lung adenocarcinoma may be due to differences in patient selection, sample number, methodology and immunohistochemical markers. 
In conclusion, D2-40-positive peritumoral LMVD may be an independent prognostic factor for lung adenocarcinoma. Detecting this indicator may predict patient prognosis in lung adenocarcinoma, while it has been suggested that reducing peritumoral lymphangiogenesis could antagonize the metastasis of lung adenocarcinoma. However, this assumption is based only on retrospective analysis of a small case series, and further experimental and clinical support with a larger number of cases is required.

\section{Acknowledgements}

We thank Manli Qi (Department of Pathology, Wuhan General Hospital of Guangzhou Command, People's Liberation Army, Wuhan, China) for her excellent technical assistance. This study was supported by the Natural Science Foundation of Hubei Province, China (no. 2010CDB09204) and the Youth Dawn Plan of Science and Technology in Wuhan, China (no. 201150431137).

\section{References}

1. Tiseo M, Bartolotti M, Gelsomino F and Ardizzoni A: First-line treatment in advanced non-small-cell lung cancer: the emerging role of the histologic subtype. Expert Rev Anticancer Ther 9: 425-435, 2009.

2. Alitalo K, Tammela T and Petrova TV: Lymphangiogenesis in development and human disease. Nature 438: 946-953, 2005.

3. Stacker SA, Farnsworth RH, Karnezis T, et al: Molecular pathways for lymphangiogenesis and their role in human disease. Novartis Found Symp 281: 38-43, 2007.

4. Mandriota SJ, Jussila L, Jeltsch M, et al: Vascular endothelial growth factor-C-mediated lymphangiogenesis promotes tumour metastasis. EMBO J 20: 672-682, 2001.

5. Stacker SA, Caesar C, Baldwin ME, et al: Vascular endothelial growth factor-D promotes the metastatic spread of cancer via the lymphatics. Nat Med 7: 186-191, 2001.

6. Schoppmann SF, Birner P, Stöckl J, et al: Tumor-associated macrophages express lymphatic endothelial growth factors and are related to peritumoral lymphangiogenesis. Am J Pathol 161: 947-956, 2002.

7. Schoppmann SF, Fenzl A, Nagy K, et al: VEGF-C expressing tumor-associated macrophages in lymph node positive breast cancer: impact on lymphangiogenesis and survival. Surgery 139: 839-846, 2006.

8. Zhang B, Wang J, Gao J, et al: Alternatively activated RAW264.7 macrophages enhance tumor lymphangiogenesis in mouse lung adenocarcinoma. J Cell Biochem 107: 134-143, 2009.

9. Renyi-Vamos F, Tovari J, Fillinger J, et al: Lymphangiogenesis correlates with lymph node metastasis, prognosis, and angiogenic phenotype in human non-small cell lung cancer. Clin Cancer Res 11: 7344-7353, 2005.

10. Takanami I: Lymphatic microvessel density using D2-40 is associated with nodal metastasis in non-small cell lung cancer. Oncol Rep 15: 437-442, 2006.

11. Kadota K, Huang CL, Liu D, et al: The clinical significance of lymphangiogenesis and angiogenesis in non-small cell lung cancer patients. Eur J Cancer 44: 105710-105767, 2008.

12. Iwakiri S, Nagai S, Katakura H, et al: D2-40-positive lymphatic vessel density is a poor prognostic factor in squamous cell carcinoma of the lung. Ann Surg Oncol 16: 1678-1685, 2009.

13. Faoro L, Hutto JY, Salgia R, et al: Lymphatic vessel density is not associated with lymph node metastasis in non-small cell lung carcinoma. Arch Pathol Lab Med 132: 1882-1888, 2008.

14. Travis WD, Brambilla E, Noguchi M, et al: International Association for the Study of Lung Cancer/American Thoracic Society/European Respiratory Society International Multidisciplinary Classification of lung adenocarcinoma. J Thorac Oncol 6: 244-285, 2011.
15. Kahn HJ and Marks A: A new monoclonal antibody, D2-40, for detection of lymphatic invasion in primary tumors. Lab Invest 82: 1255-1257, 2002.

16. Kato S, Shimoda H, Ji RC and Miura M: Lymphangiogenesis and expression of specific molecules as lymphatic endothelial cell markers. Anat Sci Int 81: 71-83, 2006.

17. Braun M, Wardelmann E, Debald M, et al: Detection of lymphovascular invasion in vulvar cancer by D2-40 (podoplanin) as a predictor for inguinal lymph node metastases. Onkologie 32: 732-738, 2009.

18. Kitano H, Kageyama S, Hewitt SM, et al: Podoplanin expression in cancerous stroma induces lymphangiogenesis and predicts lymphatic spread and patient survival. Arch Pathol Lab Med 134: 1520-1527, 2010.

19. Min KH, Park SJ, Lee KS, et al: Clinical usefulness of D2-40 in non-small cell lung cancer. Lung 189: 57-63, 2011.

20. Raica M, Cimpean AM, Ceausu R and Ribatti D: Lymphatic microvessel density, VEGF-C, and VEGFR-3 expression in different molecular types of breast cancer. Anticancer Res 31: 1757-1764, 2011.

21. Kim HS, Sung W, Lee S, Chang SG and Park YK: Lymphatic vessel densities of lymph node-negative prostate adenocarcinoma in Korea. Pathol Res Pract 205: 249-254, 2009.

22. Saad RS, Kordunsky L, Liu YL, Denning KL, Kandil HA and Silverman JF: Lymphatic microvessel density as prognostic marker in colorectal cancer. Mod Pathol 19: 1317-1323, 2006.

23. Valencak J, Heere-Ress E, Kopp T, Schoppmann SF, Kittler H and Pehamberger $\mathrm{H}$ : Selective immunohistochemical staining shows significant prognostic influence of lymphatic and blood vessels in patients with malignant melanoma. Eur J Cancer 40: 358-364, 2004

24. Ali MA: Lymphatic microvessel density and the expression of lymphangiogenic factors in oral squamous cell carcinoma. Med Princ Pract 17: 486-492, 2008.

25. Dadras SS, Paul T, Bertoncini J, et al: Tumor lymphangiogenesis: a novel prognostic indicator for cutaneous melanoma metastasis and survival. Am J Pathol 162: 1951-1960, 2003.

26. Maula SM, Luukkaa M, Grénman R, Jackson D, Jalkanen S and Ristamäki R: Intratumoral lymphatics are essential for the metastatic spread and prognosis in squamous cell carcinomas of the head and neck region. Cancer Res 63: 1920-1926, 2003.

27. Horiguchi A, Ito K, Sumitomo M, Kimura F, Asano T and Hayakawa M: Intratumoral lymphatics and lymphatic invasion are associated with tumor aggressiveness and poor prognosis in renal cell carcinoma. Urology 71: 928-932, 2008.

28. Padera TP, Stoll BR, Tooredman JB, Capen D, Di Tomaso E and Jain RK: Pathology: cancer cells compress intratumour vessels. Nature 427: 695, 2004.

29. Padera TP, Kadambi A, Di Tomaso E, et al: Lymphatic metastasis in the absence of functional intratumor lymphatics. Science 296 : 1883-1886, 2002

30. Ji RC: Lymphatic endothelial cells, tumor lymphangiogenesis and metastasis: new insights into intratumoral and peritumoral lymphatics. Cancer Metastasis Rev 25: 677-694, 2006.

31. Wang XL, Fang JP, Tang RY and Chen XM: Different significance between intratumoral and peritumoral lymphatic vessel density in gastric cancer: a retrospective study of 123 cases. BMC Cancer 10: 299, 2010.

32. Gao Y, Liu Z, Gao F and Meng XY: High density of peritumoral lymphatic vessels is a potential prognostic marker of endometrial carcinoma: a clinical immunohistochemical method study. BMC Cancer 10: 131, 2010.

33. Adachi Y, Nakamura H, Kitamura Y, et al: Lymphatic vessel density in pulmonary adenocarcinoma immunohistochemically evaluated with anti-podoplanin or anti-D2-40 antibody is correlated with lymphatic invasion or lymph node metastases. Pathol Int 57: 171-177, 2007. 